\title{
Deciphering the Environmental Impacts on Rice Quality for Different Rice Cultivated Areas
}

\author{
Xiukun $\mathrm{Li}^{\dagger}$, Lian $\mathrm{Wu}^{\dagger}$, Xin Geng, Xiuhong Xia, Xuhong Wang, Zhengjin Xu and Quan Xü (D)
}

\begin{abstract}
Background: Rice (Oryza sativa L.) is cultivated in a wide range of climatic conditions, and is one of mankind's major staple foods. The interaction of environmental factors with genotype effects major agronomic traits such as yield, quality, and resistance in rice. However, studies on the environmental factors affecting agronomic traits are often difficult to conduct because most environmental factors are dynamic and constantly changing.

Results: A series of recombinant inbred lines (RILs) derived from an indica/japonica cross were planted into four typical rice cultivated areas arranging from latitude $\mathrm{N} 22^{\circ}$ to $\mathrm{N} 42^{\circ}$. The environmental data from the heading to mature (45 days) stages were recorded for each RIL in the four areas. We determined that light, temperature, and humidity significantly affected the milling quality and cooking quality overall the four areas. Within each area, these environmental factors mainly affected the head rice ratio, grain length, alkali consumption, and amylose and protein content. Moreover, the effect of these environmental factors dynamically changed from heading to mature stage. Compared to light and humidity, temperature was more stable and predictable, and night temperature showed a stronger correlation efficiency to cooking quality than day temperature, and the daily temperature range had contrary effects compared to day and night temperature on grain quality.
\end{abstract}

Conclusions: The present study evaluated the critical phase during the grain filling stage by calculating the dynamic changes of correlation efficiency between the quality traits and climate parameters. Our findings suggest that the sowing date could be adjusted to improve rice quality so as to adjust for environmental changes.

Keywords: Rice, Environmental factors, Quality, Dynamic deciphering

\section{Background}

Grain yield and quality are major properties that are often investigated by agricultural scientists and breeders. Global climate change has also directly affected the environment and crop production. Several studies have shown that environmental factors had extreme effects on crop yield (Peng et al. 2004; Shi et al. 2016; Kim et al. 2016). As the living standards of society and the economy have significantly improved during the past decades, studies have focused on rice (Oryza sativa L.)

\footnotetext{
* Correspondence: kobexu34@live.cn

Xiukun Li and Lian Wu contributed equally to this work.

${ }^{\dagger}$ Equal contributors

Rice Research Institute of Shenyang Agricultural University, Shenyang 110866, China
}

quality traits, such as milling and cooking quality. High night temperature has been reported to decrease head rice ratio, increase chalkiness, and reduce grain width in rice (Shi et al. 2016). Rice chalkiness is a complex polygenic trait that is easily influenced by environmental conditions and certain cultural practices, particularly during the grain filling stage (Liu et al. 2010; Siebenmorgen et al. 2013). Although highly influenced by the environment, amylose content, grain length, grain width, and aspect ratio are mainly controlled by genetics (Fitzgerald et al. 2009). Poor grain quality caused by an increase in night temperature may lead to extensive reduction in economic benefits (Lyman et al. 2013).

Studies on the effects of environmental factors during the crop growth period could provide accurate information for evaluating the impact of climate on crop production. The effects of genotype, environment, and 
genotype $\times$ environment interaction determine the phenotypic performance and its general and specific adaptation to different environmental conditions (Balakrishnan et al. 2016). The genotype of plants can be determined through molecular and genomic approaches using DNA markers and high-throughput sequencing (Koboldt et al. 2013). However, the determination and measurement of environmental factors are relatively difficult and are largely due to two reasons. First, environmental factors are highly dynamic and constantly changing during the plant growth period, and the simple average or sum of all data cannot explain the complex effects of environmental factors. Second, environmental factors are nontrivial to be zoomed into specific individual plants so that the environmental data could be matched to the corresponding genotype. Dissecting various quantitative traits into individual Mendelian factors using molecular markers has accelerated the quantitative genetics walk out from the multiple gene investigation (Paterson et al. 1988; Lander and Botstein 1989). Dissecting complex environments into individual factors and measuring individual plants during the entire growth period may dramatically improve our understanding of the effects of environmental factors on crop production (Xu 2016). Because grain yield, quality, and stress resistance are complex traits that are affected by the environment, the selection of genotypes based on performance under a single environmental condition is inadequate for the elucidation of the effect of an environmental factor on crop production.
An investigation based on a multi-environment may provide more information of the effects of environmental factors on crop production.

The main objective of the present study was to dynamically and constantly decipher the effects of environmental factors on quality traits of rice under different environmental conditions.

\section{Results}

\section{Assessment of Environmental Dactors in Four Areas}

To determine the effect of various environmental factors on rice quality, we planted a series of 155 recombinant inbred lines (RILs), derived from a cross between indica variety "R99" and japonica variety "SN265" into four typical rice cultivate areas, SY $\left(\mathrm{N} 42^{\circ}\right)$, JS $\left(\mathrm{N} 32^{\circ}\right), \mathrm{SC}$ $\left(\mathrm{N} 31^{\circ}\right)$, and $\mathrm{SZ}\left(\mathrm{N} 22^{\circ}\right)$. The location of the cultivated area is shown in Fig. 1a. The survey of environmental data included light, temperature, and humidity was conducted for nearly 3 months at all four areas. Because the 155 lines have different heading dates, the environmental conditions of 45 days after heading were very different for each line (Additional file 1: Table S1). Thus, the plants underwent diverse environmental conditions from the heading stage to the mature stage even if they were cultivated in same area. In the present study, we selected the environmental data of day 1 after heading for 155 lines in SY, then used the average data to represent the environmental condition for day 1 after heading in SY. Then, we collected data from day 1 to day 45 after
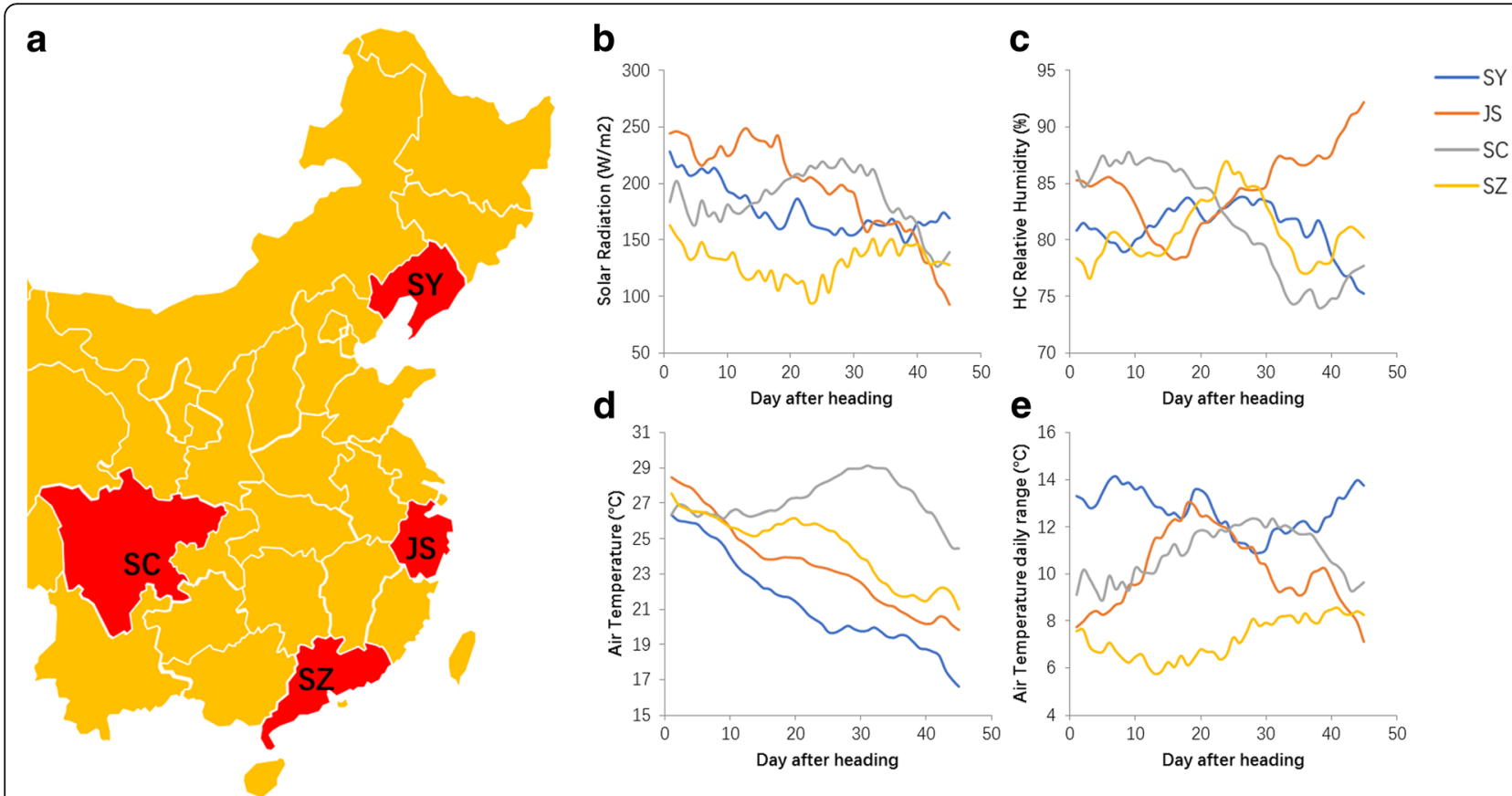

Fig. 1 The main environmental factors of the four cultivated areas. a The position of four typical rice cultivated areas, $\mathbf{b}$-e The data from 45 days of monitoring various environmental factors in four areas 
heading using this method. These data represented the environmental conditions which plants really experience from the heading stage to the mature stage in SY. The environmental data of the 45-day from heading of each line in four areas were collected to conduct the following analysis. The results showed that the environmental conditions that RILs experienced during those 45 days were highly diverse (Fig. 1). The air temperature was more stable and predictable than were light and humidity, and the air temperature was ranked as $\mathrm{SC}>\mathrm{SZ}>\mathrm{JS}>\mathrm{SZ}$ (Fig. 1d). SY had a high daily temperature range during the 45 days of monitoring, SC and JS showed a similar daily temperature range as that of SY for 20-30 days, and SZ had a low daily temperature range (Fig. 1e). In terms of solar radiation, SY and JS experienced more radiation than did $\mathrm{SC}$ and $\mathrm{SZ}$ during the earlier stage; for SC, it increased at the later stage, and SZ received the least solar radiation during the entire study (Fig. 1b). Humidity showed the opposite patterns as those observed for solar radiation (Fig. 1c).

\section{Quality Measurement of RILs in the Four Areas}

We conducted a quality measurement, including brown rice ratio, milled rice ratio, head rice ratio, chalkiness rice ratio, chalkiness level, grain length, grain width, alkali consumption, gel consistency, amylose content, and protein content of the RILs in the four areas upon reaching maturity. Our results showed extensive variations in quality traits among the four areas. All traits exhibited significant differences among the four areas except for brown rice ratio, milled rice ratio, and alkali consumption between SY and JS, and the chalkiness rice ratio, chalkiness level, and gel consistency between SC and SZ (Fig. 2 and Table 1). We subsequently compared variations that may have been caused by differences in environmental conditions and genetic diversity. The maximum data minus the minimum data of the 155 lines in one area represent the variation caused by genotype. The maximum data minus the minimum data among the four areas for an individual line represent the variation caused by environmental conditions. The results showed that the environmental conditions have a stronger effect on protein content and alkali consumption than genotype, and genotype has stronger impact on amylose content and grain width than environmental factors (Table 1).

\section{Relationship Between Environmental Factors and Rice Quality}

We subsequently divided light factor into solar radiation, lux meter, and light hour, divided air temperature into whole day average air temperature, day temperature, night temperature, and daily temperature range, and divided relative humidity into whole day average humidity, day humidity, and night humidity, generating a total of 10 parameters. Because each line had specific 45 days of environmental data, and each line also had a value of quality traits, the correlation analysis could be conducted between environmental data and quality traits. The present analysis was based on a one-on-one relationship between a specific environmental factor and particular individual line. Then, we conducted a

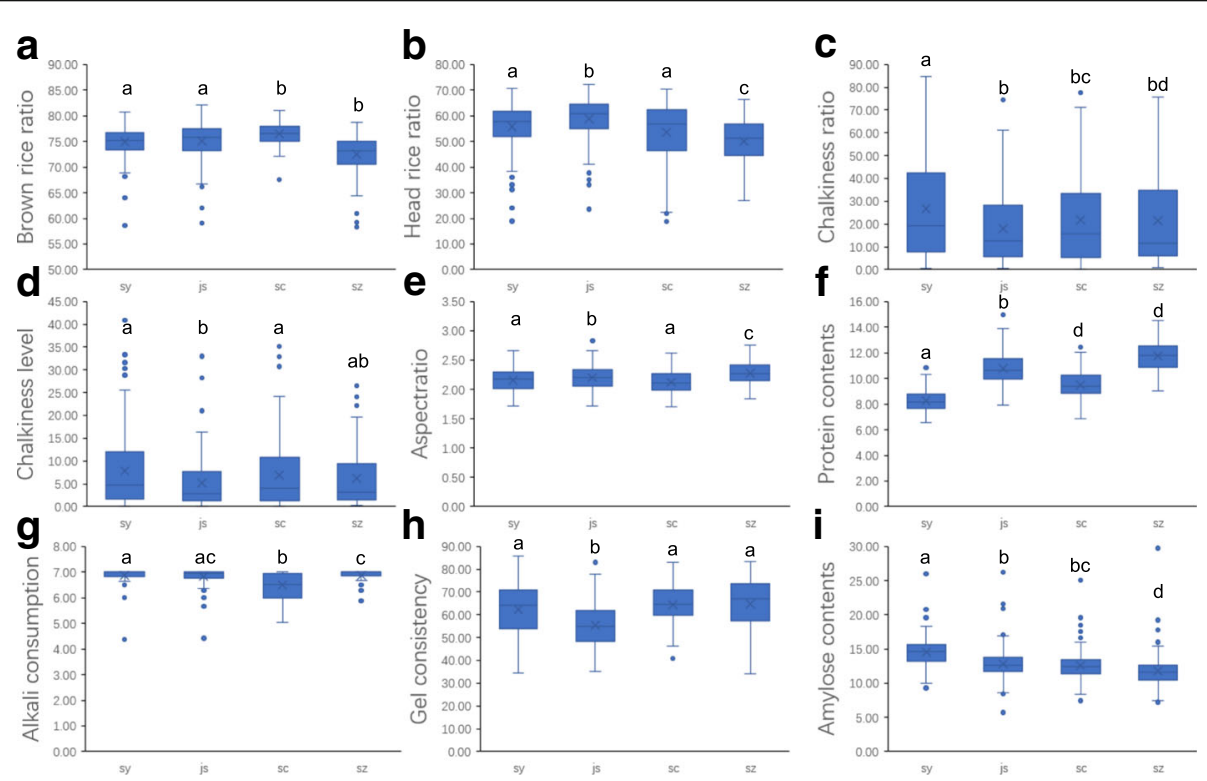

Fig. 2 The box-plot graph of the rice quality traits of RILs in four cultivated areas. a brown rice ratio, $\mathbf{b}$ head rice ratio, $\mathbf{c}$ chalkiness rice ratio, $\mathbf{d}$ chalkiness level, e grain length/grain width, $\mathbf{f}$ protein content, $\mathbf{g}$ alkali consumption, $\mathbf{h}$ gel consistency, and $\mathbf{i}$ amylose content. Letter difference means significant at 5\% probability levels by Duncan's new multiple range method 


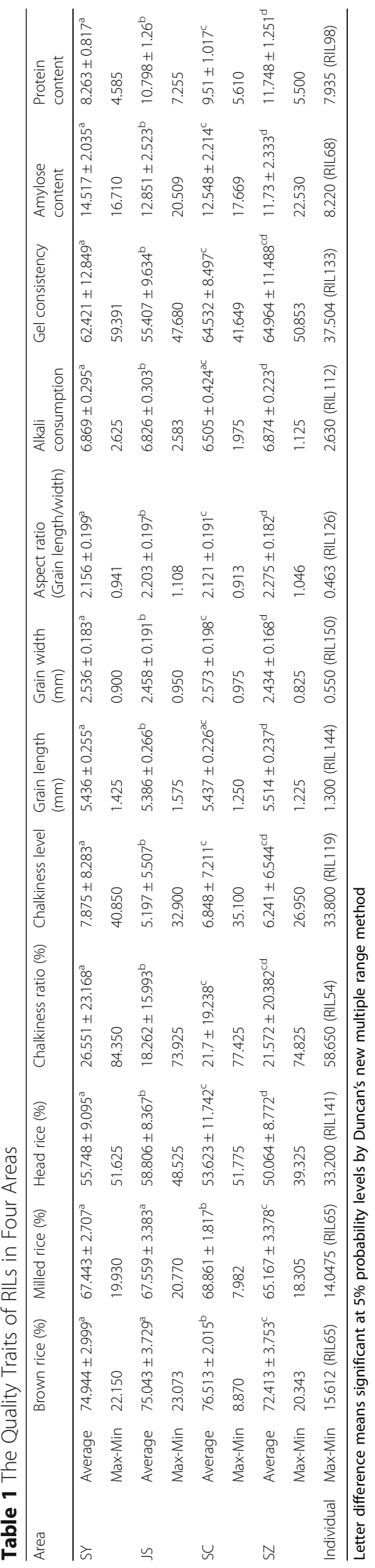


correlation analysis between quality traits and the $10 \mathrm{pa}-$ rameters. In its entirety, the 10 parameters were significantly correlated with almost all quality traits except for chalkiness traits. Temperature showed a weaker correlation with grain shape traits than did light and humidity. Within each area, these 10 parameters exhibited a weaker correlation with brown rice ratio, milled rice ratio, and gel consistency than over all four areas together. The 10 parameters showed weaker correlation with quality traits in SZ than that in the other three areas (Table 2).

Protein content showed a significant positive correlation with whole day average temperature, and the protein content of plants grown in SY, JS and SZ could be ranked as $\mathrm{SY}<\mathrm{JS}<\mathrm{SZ}$. However, $\mathrm{SC}$, which had the highest temperature, had protein content that were only higher than that of SY. This may be explained by changes in solar radiation and humidity in SC. Solar radiation increased from 10 days after heading and was highest at 20-30 days, whereas a negative correlation between solar radiation and protein content was observed in this stage. Humidity significantly decreased after 20 days and was significantly correlated with protein content during this stage. Consequently, changes in solar radiation and humidity in SC may have impaired the increase of protein content that was caused by high temperature.

In order to elucidate the interaction between each environmental factor to rice quality, we conducted a path analysis as shown in Additional file 1: Table S2. For milling quality, the indirect path coefficients from solar radiation through daily temperature range to brown rice ratio and milled rice ratio were 0.423 and 0.399 , respectively. The indirect path coefficients from solar through daily temperature range were 0.423 and 0.399 , respectively. For appearance quality, the indirect path coefficient from solar through daily temperature range was -0.245 . For cooking quality, the indirect path coefficients from solar through daily temperature range to alkali consumption and amylose content were -0.236 and 0.422 , respectively. For nutritional quality, the indirect path coefficients from solar through daily temperature range to protein content was -0.843 . For amylose content and protein content, the indirect path coefficient from relative humidity through solar radiation also reached -0.236 and 0.335 , respectively. The indirect path coefficients among environmental factors were at a low level in head rice ratio and gel consistency.

Dynamic analysis of Environmental Factors to Rice Quality As environmental factors are dynamic and constantly change, we conducted a dynamic analysis of the environmental factors on quality to elucidate the effects of changes in environmental factors from the heading stage to the mature stage. The results showed that the effect of environmental factors became stronger or weaker over time (Fig. 3). Compared to light and humidity, the effect of temperature on rice quality traits was more stable and predictable. The whole day average temperature showed a significant positive correlation with protein content and a significant negative correlation with amylose content, alkali consumption, and head rice ratio during the entire 45-day survey; it had a negative correlation with brown rice ratio in the first 20 days, but subsequently exhibited a positive correlation (Fig. 3). Interestingly, the daily temperature range exhibited the opposite effects compared to the whole day average temperature, particularly in terms of protein content, amylose content, grain length, and head rice ratio (Fig. 4). Day temperature and night temperature showed similar effects as that of whole day average temperature, whereas the night temperature exhibited a stronger correlation with protein content, amylose content, and head rice ratio. The effects of light and humidity on quality traits showed weaker regularity compared to temperature. Solar radiation exhibited a negative correlation with protein content, alkali consumption, and grain length during the entire 45-day survey. Solar radiation was found to have a positive correlation with brown rice ratio and head rice ratio during the early and middle stages, yet a negative correlation was observed at the later stage. Lux meter and light hour showed similar effects on quality traits as that of solar radiation (Additional file 1: Figure S1). Day and night humidity also exhibited similar correlations with rice quality traits as that of whole day average humidity (Additional file 1: Figure S2).

\section{Discussion}

Agronomic traits such as yield, quality and stress resistance are mainly determined by the interaction between genotype and environment. Genotypes are generally assessed using DNA markers and high-throughput sequencing (Koboldt et al. 2013). Measuring the impact of the environment has largely been considered as a single factor that interacts with the particular genotype of a specific plant. As plants grow in a constantly changing environment, plants with different growth periods are subjected to variations in environmental conditions even when they are cultivated in the same area. Thus, investigations on the impact of environmental factors on crop production of specific plants are warranted.

The previous studies showed that environmental factors strongly affect rice quality. In the filling stage, the head rice ratio decreased $24 \%-35 \%$ when the temperature increased from 20 to $30{ }^{\circ} \mathrm{C}$ (Ha et al. 1994). The air temperature has been reported to have a negative correlation with amylose content (Asaoka et al. 1985), and was the major factor effecting protein content in 


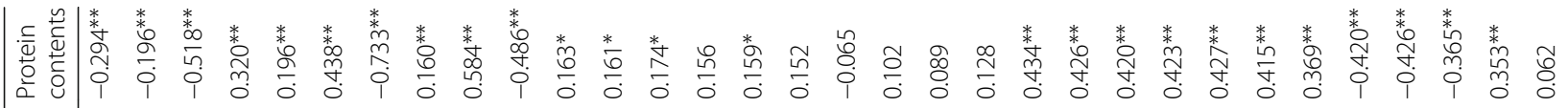

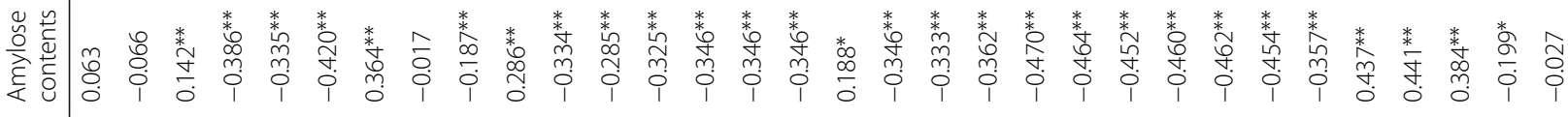

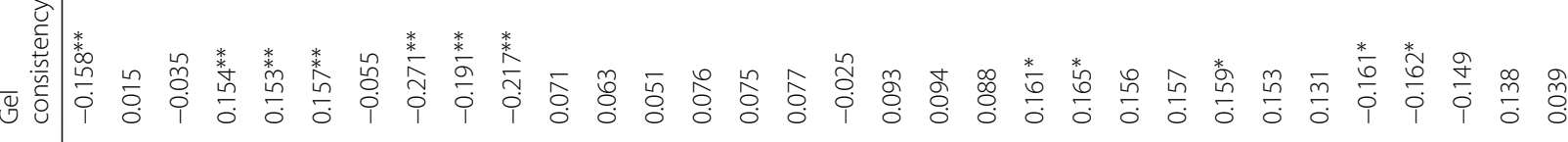

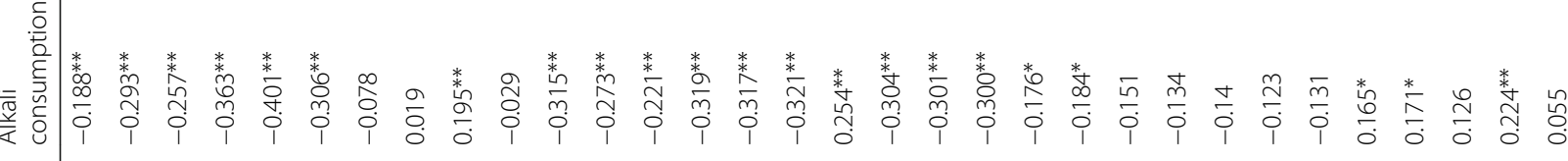

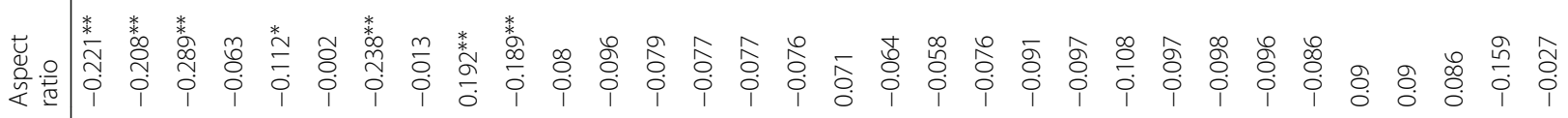

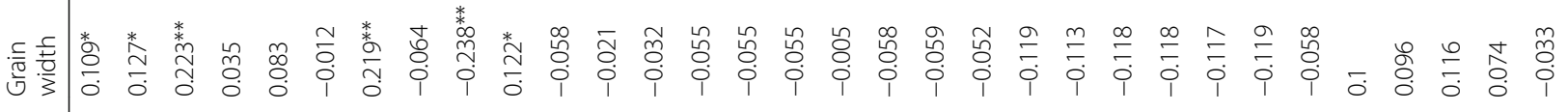

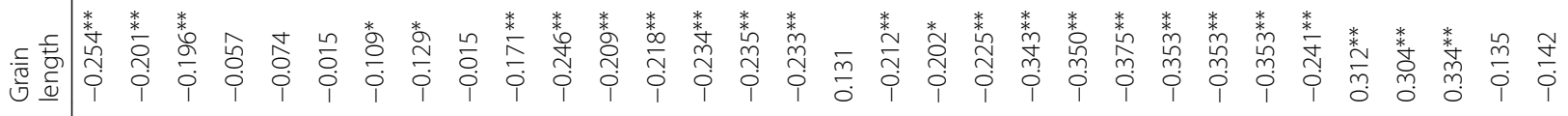

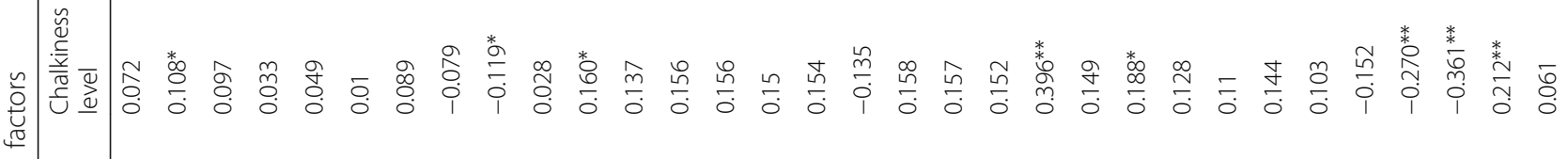

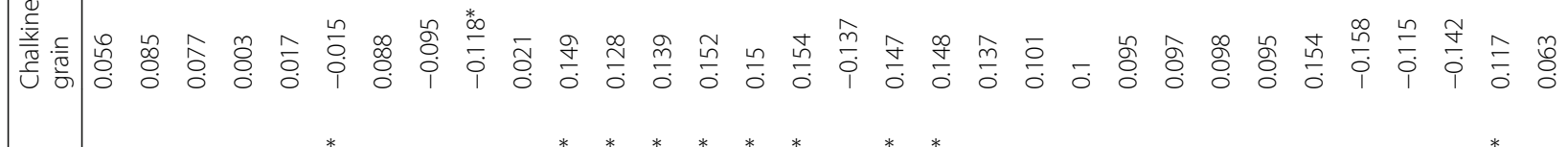

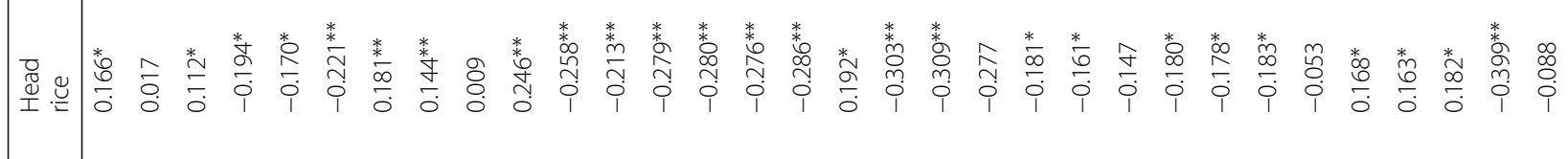

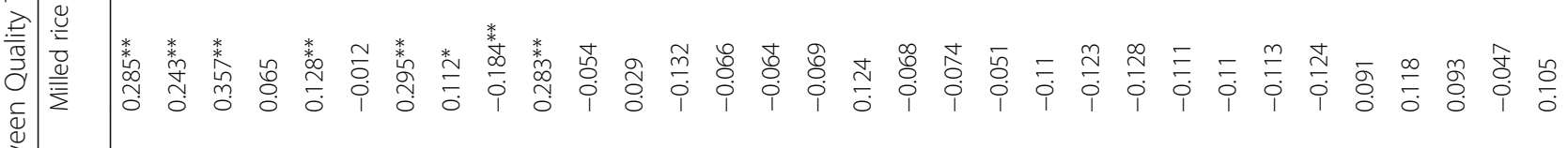

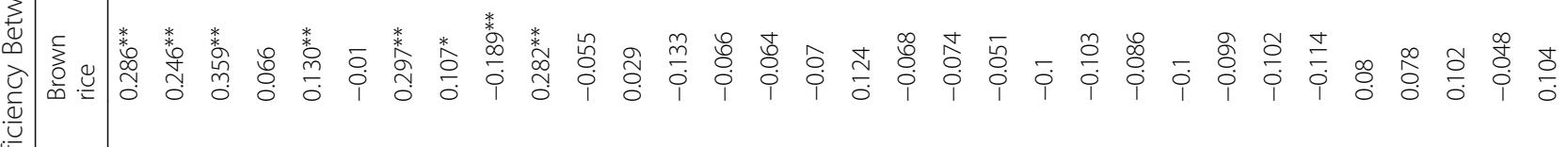

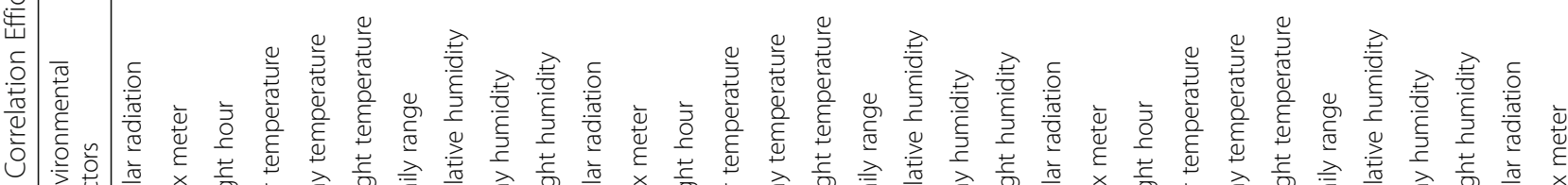

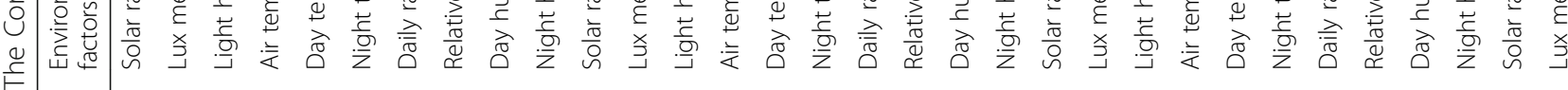
竞 


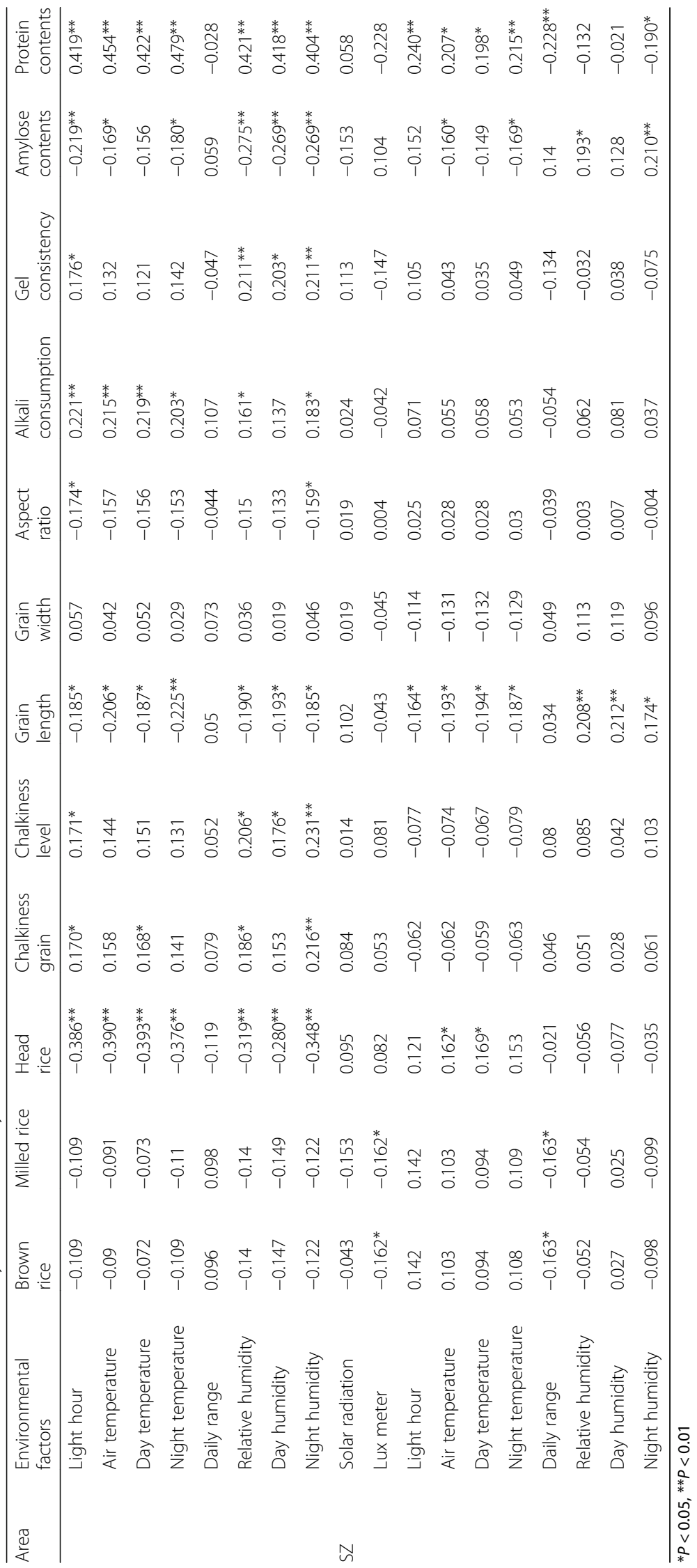



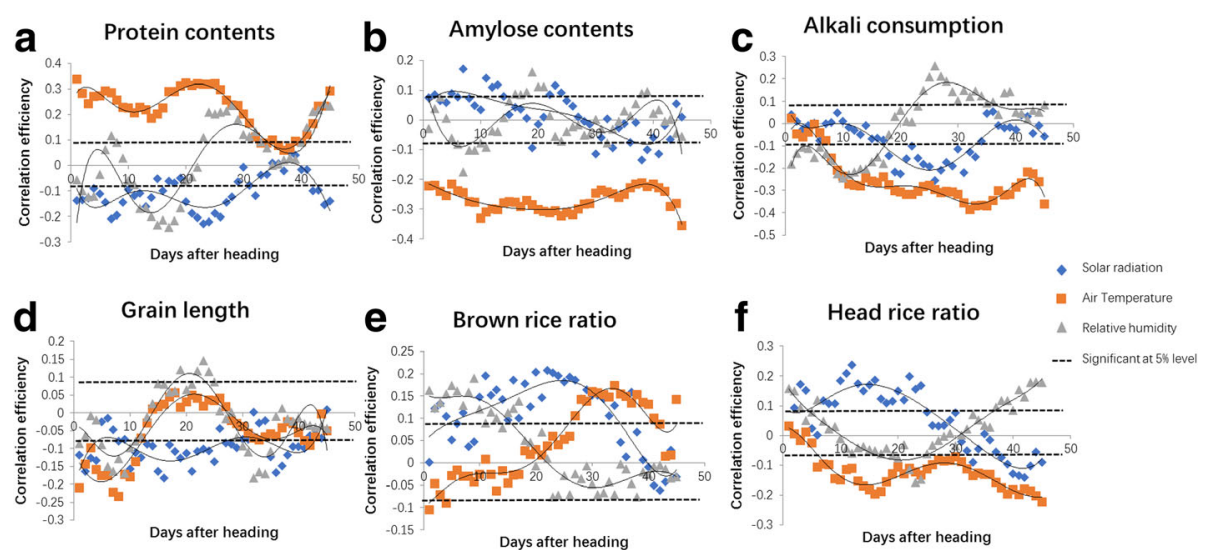

Fig. 3 The dynamic analysis of the correlation of environmental traits to quality traits. The correlation efficiency of environmental factors (solar radiation, whole day average temperature, and relative humidity) to $\mathbf{a}$ protein content, $\mathbf{b}$ amylose content, $\mathbf{c}$ alkali consumption, $\mathbf{d}$ grain length, e brown rice ratio, and $\mathbf{f}$ head rice ratio

rice during the period of 10-20 days after heading (Nakamura et al. 1989). However, few studies simultaneously investigated the effects of solar radiation, temperature and humidity on rice milling quality, appearance quality, nutritional quality and cooking quality. The present study integrated the data of solar radiation, air temperature, daily temperature range and relative humidity, with the goal of elucidating the interaction between each environmental factor and RIL lines on rice quality. The path analysis results showed that solar radiation and daily temperature range have a significant correlation with almost all quality traits. Also, the indirect path coefficient from solar radiation through daily temperature range to quality traits were at a high level. For amylose content and protein content, the indirect path coefficient from solar radiation through relative humidity also reached a high level. These results indicated that solar radiation and daily temperature range may have an interaction, and play a major role in effecting quality traits. The relative humidity may participate in this interaction to affect amylose content and protein content. Moreover, the present study determined that the effects of environmental factors on quality traits dramatically change from the heading to the mature stage. For example, the air temperature was negatively correlated with the brown rice ratio before 20 days, but was significantly positively correlated after 20 days. Humidity showed a significant negative correlation with head rice ratio in the mid stage, whereas a significant positive correlation with head rice ratio was observed at the earlier and later stages. In summary, environmental factors are dynamic and constantly changing; therefore, simply calculating the average data of environmental factors may not precisely explain their effects on quality traits. The results of our
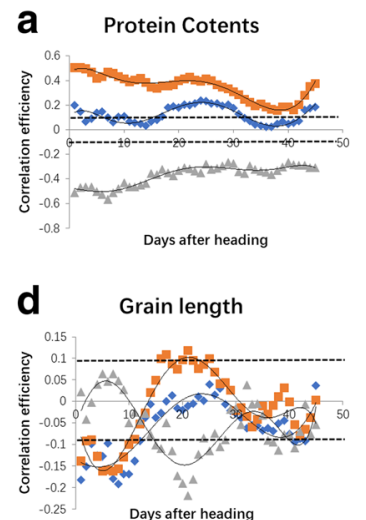
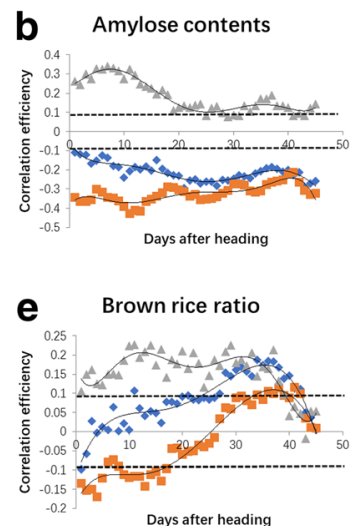
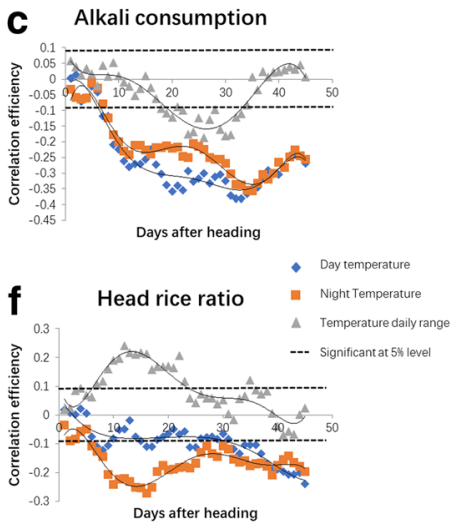

Fig. 4 The dynamic analysis of the correlation of temperature factors to quality traits. The correlation efficiency of temperature factors (day average temperature, night average temperature, and daily temperature range) to a protein content, $\mathbf{b}$ amylose content, $\mathbf{c}$ alkali consumption, $\mathbf{d}$ grain length, e brown rice ratio, and $\mathbf{f}$ head rice ratio 
study suggest that the appropriate adjustments to the sowing date should be made to cater to or avoid to the corresponding advantages or disadvantages of specific environmental conditions.

An investigation of the years from 1951 to 2010 has observed an asymmetric warming, with greater increase in night than in day temperature (Donat and Alexander 2012). The annual mean day temperature and night temperature increased by $0.35{ }^{\circ} \mathrm{C}$ and $1.13{ }^{\circ} \mathrm{C}$, respectively (Peng et al. 2004). Such an increase in air temperature will profoundly affect crop production, and many studies show a significant influence of elevated temperature on crop yield. Grain yield declined by $10 \%$ for each $1{ }^{\circ} \mathrm{C}$ increase (Peng et al. 2004). Grain quality in most hybrids was also strongly affected by high night temperature, decreased head rice ratio, increased chalkiness, and reduced grain width (Shi et al. 2016). A metaanalysis demonstrated that high temperature affected grain quality by decreasing the head rice percentage and increasing the chalkiness rice ratio and chalkiness level (Xiong et al. 2017). In our study, we also found that night temperature more severely affected quality traits than day temperature. Moreover, we found that the daily temperature range is an important parameter that affects quality as it has the opposite effect on quality traits compared to day and night temperature. The whole day average temperature has a significant negative correlation with amylose content, but the daily temperature range showed a positive correlation with amylose content. The lowest whole day average temperature and the largest daily temperature range in SY resulted in the highest amylose content. The smallest temperature range in $\mathrm{SZ}$ resulted in the lowest amylose content despite the lower whole day average temperature relative to that of SC.

The environmental factors strongly affected the yield components. However, we only conducted a 45-day survey of the environmental factors, and thus, we were unable to evaluate the effect of environmental factors on heading date, plant height, and various yield components. The effect of various environmental factors on the entire growth period should be investigated in a future study. We found that some RILs showed extremely large variations among four areas, whereas only a few RILs exhibited a stable phenotype in all four areas. The molecular mechanism underlying the stability of a phenotype among different areas requires further investigation. The stable lines may also serve as a rare germplasm for breeding highly adaptable varieties that exhibit elite phenotypic features in different areas.

\section{Conclusions}

The precise dissection of complex environmental factors for specific individual plants provides us with a novel strategy for the optimization of environmental factors for crop quality improvement. In the present study, we monitored various environmental factors surrounding 155 RILs that were planted in four areas for 45 days to decipher their impact on rice grain quality. The results showed that environmental factors had variable effects on rice grain quality, ranging from the heading stage to the mature stage. Based on our findings, we suggest that field management adjust the sowing date to cater to the current environmental conditions in order to improve rice quality.

\section{Method \\ Plant Materials}

A total of 155 RILs derived from a cross between 'Shennong265' (Oryza. sativa L. ssp. japonica) and 'R99' (Oryza. sativa L. ssp. indica) was used in this study. This RIL population was developed from a single-seed descendant that had been inbred for over 10 generations. Field experiments were conducted in four typical rice cultivated areas: the Rice Research Institute of Shenyang Agricultural University (SY) $\left(\mathrm{N} 41^{\circ}, \mathrm{E} 123^{\circ}\right)$, the sub base of China National Hybrid Rice R\&D Center in Jiangsu Province (JS N32 ${ }^{\circ}$ E120 $0^{\circ}$, the Academy of Agricultural Sciences of Sichuan Province (SCN32 E104 ${ }^{\circ}$ ), and the Agricultural Genomics Institute at Shenzhen $\left(\mathrm{SZN} 22^{\circ}, \mathrm{E} 114^{\circ}\right)$ for two growing seasons during 2015-2016. At SY, the seeds were sown on April 15 and transplanted on May 21. At JS, they were sown on May 8 and transplanted on June 8 . At SC, they were sown on April 12 and transplanted on May 9. At SZ, they were sown on July 20 and transplanted on August 12. Seeds were sown in both 2015 and 2016. Three rows were planted for each line, with 10 plants per row, and a plant spacing of $30 \mathrm{~cm} \times 13.3 \mathrm{~cm}$. The RILs were arranged in a randomized block design with two replicates. Cultivation methods and field management varied according to regional cultivation practices. The paddies were tested before sowing, and the fertility of soil in the four areas is shown in Additional file 1: Table S3. Fertilizers were applied with a basal dressing amount of $150 \mathrm{~kg} \mathrm{~N}$ per hectare, $150 \mathrm{~kg} \mathrm{P}$ per hectare and $150 \mathrm{~kg}$ $\mathrm{K}$ per hectare. We added $75 \mathrm{~kg} \mathrm{~N}$ per hectare 7 days after transplanting as a top application. The paddies were harvested at 45 days after heading for each line in each of the four areas.

\section{Quality Measurements}

Mature rice grains were milled after harvest, air dried, and stored at room temperature for 3 months. A total of 20 plants from the middle rows were harvested for each line. The brown rice ratio, milled rice ratio, and head rice ratio were calculated after harvest. The grains were dehulled to produce brown rice using Rubber Roll 
Sheller (THU testing hunsker, Satake, Hiroshima, Japan), and brown rice ratio was determined. Thereafter, the brown rice was milled with rice-polishing machine (TM05 test mill, Satake). After milling, head rice and broken rice were separated and finally, the milled rice ratio and head rice ratio were expressed as percentage of total weight of rough rice. Amylose content and gel consistency were assessed according to The National Standard of the People's Republic of China (GB/T17891-1999). Extraction and measurement of rice protein compositions was performed as described by Li et al. (2000) and Tan et al. (1999). All samples were analyzed twice. As the 2 years of data are highly correlated and showed similar trends the 2016 data was used in the subsequent analysis (Additional file 1: Table S4).

\section{Assessment of Environmental Factors and Data Analysis}

The environmental factors were measured using iMETOS (Pessl Instruments GmbH, Weiz, Austria) at four areas. We surveyed three main environmental factors: light, which was subsequently divided into solar radiation, lux meter, and light hours; temperature, which includes whole day average air temperature, day air temperature, night air temperature, daily and temperature range; and humidity, which includes whole day average relative humidity, day relative humidity, and night humidity. The survey was conducted for nearly 3 months at all four areas. The environmental data of the 45-day from heading for each line in four areas were collected to conduct the analysis. The data were analyzed using SPSS 17.0.

\section{Additional File}

Additional file 1: Figure S1. The dynamic analysis of the correlation of light factors to quality traits. The correlation efficiency of light factors (solar radiation, Lux meter, and light hours) to (a) protein content, (b) amylose content, (c), alkali consumption (d) grain length, (e) brown rice ratio, and $(f)$ head rice ratio. Figure S2. The dynamic analysis of the correlation of humidity factors to quality traits. The correlation efficiency of humidity factors (day average humidity and night average humidity) to (a) protein content, (b) amylose content, (c) alkali consumption, (d) grain length, (e) brown rice ratio, and (f) head rice ratio. Table S1. The days to heading after sowing of 155 lines at four areas in 2015 and 2016. Table S2. The fertility of soil in four areas. Table S3. The data of rice quality in 2015 and 2016. Table S4. Path analysis of the effect of environmental factors on rice quality. (PPTX $472 \mathrm{~kb}$ )

\section{Acknowledgments}

The National Natural Science Foundation of China (31430062) supported this study.

\section{Authors' Contributions}

ZX and QX designed the research plan; $X L$ and $L W$ performed most of the experiments. XG and XX participated in the assessment of environmental factors in four areas. XW participated in quality measurement; QX wrote the paper. All authors read and approved the final manuscript.

\section{Competing Interests}

The authors declare that they have no competing interests.

\section{Publisher's Note}

Springer Nature remains neutral with regard to jurisdictional claims in published maps and institutional affiliations.

Received: 13 September 2017 Accepted: 2 January 2018

Published online: 19 January 2018

\section{References}

Asaoka M, Okuno K, Fuwa H (1985) Effect of environmental temperature at the milky stage on Amylose content and fine structure of Amylopectin of waxy and nonwaxy endosperm starches of Rice (L.) Agric Biol Chem 49(2):373-379

Balakrishnan D, Subrahmanyam D, Badri J, Raju AK, Rao W, Beerelli K, Mesapogu S, Surapaneni M, Ponnuswamy R, Padmavathi G, Babu VR, Neelamraju S (2016) Genotype $x$ environment interactions of yield traits in backcross introgression lines derived from Oryza sativa cv. Swarna/Oryza nivara. Front Plant Sci 7:1530. https://doi.org/10.3389/fpls.2016.01530

Donat MG, Alexander LV (2012) The shifting probability distribution of global daytime and night-time temperatures. Geophys Res Lett 39(14):132-151

Fitzgerald MA, McCouch SR, Hall RD (2009) Not just a grain of rice: the quest for quality. Trends Plant Sci 14(3):133-139

Ha KY, Lee JK, Lee SY, Lee JS (1994) Grain quality characteristics for brewing in rice. Korean J Crop Sci 39(1):38-44

Kim J, Sang W, Shin P, Cho H, Seo M, Yoo B, Kim KS (2016) Evaluation of regional climate scenario data for impact assessment of climate change on rice productivity in Korea. J Crop Sci Biotechnol 18(4):257-264. https://doi.org/10. 1007/s12892-015-0103-z

Koboldt DC, Steinberg KM, Larson DE, Wilson RK, Mardis ER (2013) The nextgeneration sequencing revolution and its impact on genomics. Cell 155(1): 27-38. https://doi.org/10.1016/j.cell.2013.09.006

Lander ES, Botstein D (1989) Mapping mendelian factors underlying quantitative traits using RFLP linkage maps. Genetics 121(1):185-199

Li HS (2000) Principle and technology of plant physiology and biochemistry experiment. Higher education press, pp. 184-185, 192-94.

Liu X, Guo T, Wan X, Wang H, Zhu M, Li A, Su N, Shen Y, Mao B, Zhai H, Mao L, Wan J (2010) Transcriptome analysis of grain-filling caryopses reveals involvement of multiple regulatory pathways in chalky grain formation in rice. BMC Genomics 11:730. https://doi.org/10.1186/1471-2164-11-730

Lyman NB, Jagadish KS, Nalley LL, Dixon BL, Siebenmorgen T (2013) Neglecting rice milling yield and quality underestimates economic losses from hightemperature stress. PLoS One 8(8):e72157

Nakamura Y, Yuki K, Park SY, Ohya T (1989) Carbohydrate metabolism in the developing endosperm of Rice grains. Plant Cell Physiol 30(6):833-839

Paterson AH, Lander ES, Hewitt JD, Peterson S, Lincoln SE, Tanksley SD (1988) Resolution of quantitative traits into Mendelian factors by using a complete linkage map of restriction fragment length polymorphisms. Nature 335(6192): 721-726. https://doi.org/10.1038/335721a0

Peng S, Huang J, Sheehy JE, Laza RC, Visperas RM, Zhong X, Centeno GS, Khush GS, Cassman KG (2004) Rice yields decline with higher night temperature from global warming. Proc Natl Acad Sci U S A 101(27):9971

Shi W, Yin X, Struik PC, Xie F, Schmidt RC, Jagadish KSV (2016) Grain yield and quality responses of tropical hybrid rice to high night-time temperature. Field Crop Res 190:18-25

Siebenmorgen TJ, Grigg BC, Lanning SB (2013) Impacts of preharvest factors during kernel development on rice quality and functionality. Annu Rev Food Sci Technol 4:101-115. https://doi.org/10.1146/annurev-food-030212-182644

Tan YF, Li JX, Yu SB, Xing YZ, Xu CG, Zhang Q (1999) The three important traits for cooking and eating quality of rice grains are controlled by a single locus in an elite rice hybrid, Shanyou 63. Theoretical \& Applied Genetics 99(4):642-48.

Xiong D, Ling X, Huang J, Peng S (2017) Meta-analysis and dose-response analysis of high temperature effects on rice yield and quality. Environ Exp Bot 141:1-9

Xu Y (2016) Envirotyping for deciphering environmental impacts on crop plants. Theor Appl Genet 129(4):653-673 